This item was submitted to Loughborough's Research Repository by the author.

Items in Figshare are protected by copyright, with all rights reserved, unless otherwise indicated.

\title{
On the origin of heavy-tail statistics in equations of the Nonlinear Schrodinger type
}

PLEASE CITE THE PUBLISHED VERSION

http://dx.doi.org/10.1016/j.physleta.2016.07.048

PUBLISHER

(c) Elsevier

VERSION

AM (Accepted Manuscript)

\section{PUBLISHER STATEMENT}

This work is made available according to the conditions of the Creative Commons Attribution-NonCommercialNoDerivatives 4.0 International (CC BY-NC-ND 4.0) licence. Full details of this licence are available at: https://creativecommons.org/licenses/by-nc-nd/4.0/

\section{LICENCE}

CC BY-NC-ND 4.0

\section{REPOSITORY RECORD}

Onorato, Miguel, Davide Proment, Gennady El, Stephane Randoux, and Pierre Suret. 2019. "On the Origin of Heavy-tail Statistics in Equations of the Nonlinear Schrodinger Type". figshare.

https://hdl.handle.net/2134/22194. 


\title{
On the origin of heavy-tail statistics in equations of the Nonlinear Schrödinger type
}

\author{
Miguel Onorato ${ }^{\mathrm{a}, \mathrm{b}}$, , Davide Proment ${ }^{\mathrm{c}}$, Gennady El $^{\mathrm{d}}$, Stephane Randoux ${ }^{\mathrm{e}}$, Pierre Suret $^{\mathrm{e}}$ \\ ${ }^{a}$ Dipartimento di Fisica, Università degli Studi di Torino, 10125 Torino, Italy \\ ${ }^{b}$ Istituto Nazionale di Fisica Nucleare, INFN, Sezione di Torino, 10125 Torino, Italy \\ ${ }^{c}$ School of Mathematics, University of East Anglia, Norwich Research Park, Norwich, NR4 7TJ, United Kingdom \\ ${ }^{d}$ Department of Mathematical Sciences, Loughborough University, Loughborough Leicestershire, LE11 3TU, United Kingdom \\ ${ }^{e}$ Laboratoire de Physique des Lasers, Atomes et Molecules, Université de Lille, UMR-CNRS 8523, France
}

\begin{abstract}
We study the formation of extreme events in incoherent systems described by the Nonliner Schrödinger type of equations. We consider an exact identity that relates the evolution of the normalized fourth-order moment of the probability density function of the wave envelope to the rate of change of the width of the Fourier spectrum of the wave field. We show that, given an initial condition characterized by some distribution of the wave envelope, an increase of the spectral bandwidth in the focusing/defocusing regime leads to an increase/decrease of the probability of formation of rogue waves. Extensive numerical simulations in 1D+1 and $2 \mathrm{D}+1$ are also performed to confirm the results.
\end{abstract}

\section{(C) 2011 Published by Elsevier Ltd.}

Keywords: Rogue waves, Freak waves, Nonlinear Schrödinger

\section{Introduction}

Processes that lead to the formation of heavy tails $[1,2,3]$ in the Probability Density Function (PDF) are of wide interest in many physical contexts $[4,5,6,7,8,9,10]$. It is well known that in homogeneous conditions, if the central limit theorem applies, a linear wave dispersive system characterized by a large number of incoherent waves is described by a Gaussian statistics; in the latter situation extreme events can still appear but they are very rare, and their probability of appearance can be derived exactly, [11, 12]. In the field of ocean waves and nonlinear optics, it has been established that the presence of nonlinearity on top of dispersion can lead to changes in the statistical properties of the system. Often rogue waves and the associated large tails in the PDF can be observed in experiments or numerical simulations from an initially incoherent wave field [13, 14]; lower probability of extreme events than the Gaussian predictions can also be encountered $[15,16]$. In all those cases the nonlinearity plays a key role in creating correlations among modes that ultimates in a deviations from Gaussian statistics.

In this Letter we present a very simple relation which can be derived from a family of universal nonlinear dispersive partial differential equations that allows one to relate the changes in the statistical properties of the wave field to the changes of its Fourier spectrum. Specifically, our focus is on the normalized fourth-order moment of the PDF which measures the relevance of the tails of the distribution with respect to the core. Large values of such moment imply the presence of heavy tails in the distribution and higher probability of extreme events. We show analytically, without 
any approximation, that an increase of the spectral bandwidth results in an increase/decrease of extreme events in focusing/defocusing regime. Here, we will first discuss the 1D+1 integrable Nonlinear Schrödinger (NLS) equation problem and then we will extend the result to non-integrable NLS type of equation in $2 \mathrm{D}+1$ and confirm our results with extensive numerical simulations.

\section{One-dimensional propagation}

The NLS equation is a universal model for describing nonlinear dispersive waves. For the present discussion, we will consider the NLS equation written as follows:

$$
i \frac{\partial A}{\partial x}=\beta \frac{\partial^{2} A}{\partial t^{2}}+\alpha|A|^{2} A,
$$

where $\alpha$ and $\beta$ are two constant coefficients that depend on the physical problem considered. If $\alpha \beta>0$ then the equation is known to be of focusing type, while if $\alpha \beta<0$ the equation is defocusing. Note that equation (1) is written as an evolution equation in space rather than in time; this notation is common in nonlinear optics and it is also suitable in hydrodynamics for describing the evolution of waves in wave tank experiments. The general problem that one wish to answer is the following: given an incoherent time series characterized by some statistical properties at one boundary of the domain, what is the PDF of the intensity of the wave field along the tank or along the fiber? Will rogue waves appear? We stress that our goal here is not to establish the validity of the NLS equation in a specific field but to highlight a fundamental mechanism that leads to the formation of extreme or rogue waves.

We start by the definition of the normalized fourth-order moment of the NLS variable $|A|$ :

$$
\kappa=\frac{\left\langle|A|^{4}\right\rangle}{\left\langle|A|^{2}\right\rangle^{2}}=\frac{\int|A|^{4} P(|A|) d|A|}{\left(\int|A|^{2} P(|A|) d|A|\right)^{2}},
$$

where $P(|A|)$ is the probability density function of the wave envelope $|A|$ and $\langle\ldots\rangle$ denotes the expected value. By definition $\kappa$ weights the relevance of tails of the PDF. Our work is based on the (now trivial) observation that the nonlinear part of the Hamiltonian is strictly related to $\kappa$. Indeed, we consider the following Hamiltonian density which is conserved for equation (1):

$$
H=\frac{1}{T} \int_{0}^{T} \beta\left|\frac{\partial A}{\partial t}\right|^{2} d t-\frac{1}{T} \int_{0}^{T} \frac{\alpha}{2}|A|^{4} d t .
$$

We then apply the expected value operator on the above equation to get:

$$
\langle H\rangle=\frac{1}{T} \int_{0}^{T} \beta\left\langle\left|\frac{\partial A}{\partial t}\right|^{2}\right\rangle d t-\frac{1}{T} \int_{0}^{T} \frac{\alpha}{2}\left\langle|A|^{4}\right\rangle d t .
$$

${ }_{41}$ (the last equality holds for a statistical stationary process) and

$$
\Omega(x)=\sqrt{\frac{\sum_{n}\left\langle\left(\frac{2 \pi}{T} n\right)^{2}\left|A_{n}(x)\right|^{2}\right\rangle}{\sum_{n}\left\langle\left|A_{n}(x)\right|^{2}\right\rangle}},
$$

with $A_{n}(x)$ being the Fourier coefficients defined as

$$
A_{n}(x)=\frac{1}{T} \int_{0}^{T} A(x, t) e^{-i \frac{2 \pi}{T} n t} d t .
$$




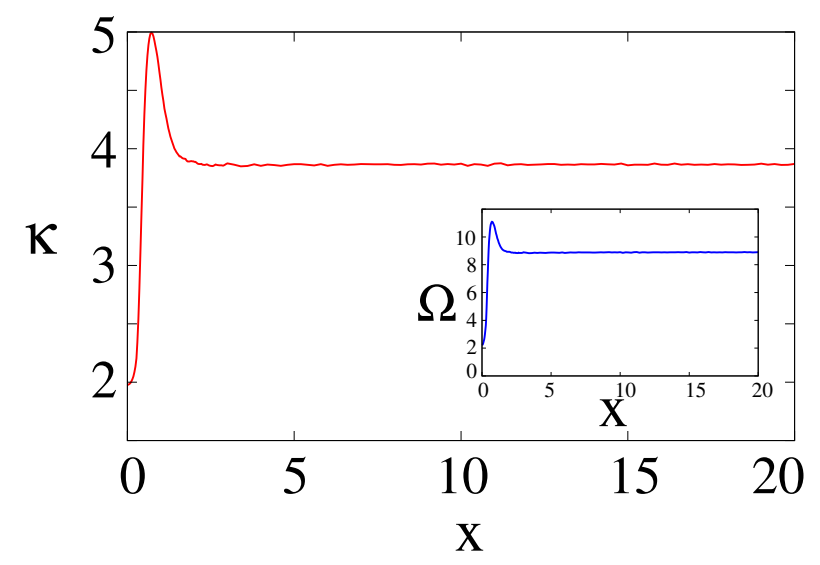

Figure 1. Evolution of $\kappa$ for the focusing NLS equation $(\alpha=2, \beta=1)$. In the inset the evolution of the spectral bandwidth is shown.

Note that periodic boundary conditions in $t$ have been assumed in order to write the Fourier series. The quantity $\Omega(x)$ defined by Eq. (7) is nothing but the definition of the spectral bandwidth (see also [17]). Evaluating the expression (5) at $x=x_{0}$ and at a generic point $x$, after eliminating $\langle H\rangle /\langle N\rangle$ from the two resulting equations, we get the following exact relation (note that $\langle H\rangle$ and $\langle N\rangle$ do not depend on space and time):

$$
\kappa(x)=\kappa\left(x_{0}\right)+2 \frac{\beta}{\alpha} \frac{1}{\langle N\rangle}\left[\Omega(x)^{2}-\Omega\left(x_{0}\right)^{2}\right] .
$$

The invariance of the Hamiltonian of the NLS equation has also been used in [18] to derive an approximate expression that relates the spectral bandwidth to the amplitude of the highest wave during the evolution of a deterministic wave group. Equation (9) implies that the variation of the fourth-order moment is directly related to the variation of the spectral bandwidth. From it, we can state that in the focusing regime, $\beta / \alpha>0$, an increase of the spectral bandwidth leads to an increase of fourth-order moment; therefore, we expect to observe more extreme or rogue waves. On the other side, in the defocusing regime, $\beta / \alpha<0$, the same increase of the spectral bandwidth is accompanied by a decrease of $\kappa$.

In what follows, we consider a few numerical examples that emphasize the above results; without loss of generality, we solve the NLS equation (1) with $\alpha= \pm 2$ and $\beta=1$, starting from an initial condition characterized by the following frequency Fourier spectrum:

$$
A_{n}(x=0)=\sqrt{a_{0} e^{-\frac{4 \pi^{2} n^{2}}{T^{2} \sigma^{2}}}} e^{i \phi_{n}}
$$

where the phases $\phi_{n}$ are distributed uniformly in the $[0,2 \pi)$ interval. The numerical simulations are performed by using a pseudo-spectral method with 4096 points. The numerical values of $a_{0}$ and $\sigma^{2}$ are 1.129 and $10^{4}$, respectively. The statistical properties of the random wave fields are computed from an ensemble of $10^{4}$ realisations of the random initial condition. Because of the latter choice, the PDF of the real and imaginary part of $A(t, x=0)$ are Gaussian, the PDF of $|A(t, x=0)|$ is distributed according to the Rayleigh distribution having $\kappa=2$, and the PDF of the intensity $|A(t, x=0)|^{2}$ is exponential. In Figures 1 and 2 we show $\kappa$ and the spectral bandwidth $\Omega$ as a function of the evolution variable $x$ for the focusing, $\alpha=2$, and the defocusing case, $\alpha=-2$, respectively. It is interesting to note that, regardless of the sign of $\alpha$, the spectral bandwidth always increases; however, while $\kappa$ increases in the focusing case, it decreases in the defocusing one. As was mentioned, high values of $\kappa$ implies heavy tails in the PDF. Indeed, in Figure 3 the PDF of the normalized intensity $I=|A(t, x)|^{2} / N$ computed after $\kappa$ has reached an equilibrium state, $(x>20)$, is shown for both the focusing and the defocusing case. Numerical results are compared with the exponential distribution $e^{-I}$; deviations from such distribution are observed for both cases, however, consistently with our derivation, the focusing case shows heavy tails, while in the defocusing one, the distribution is below the exponential prediction. 


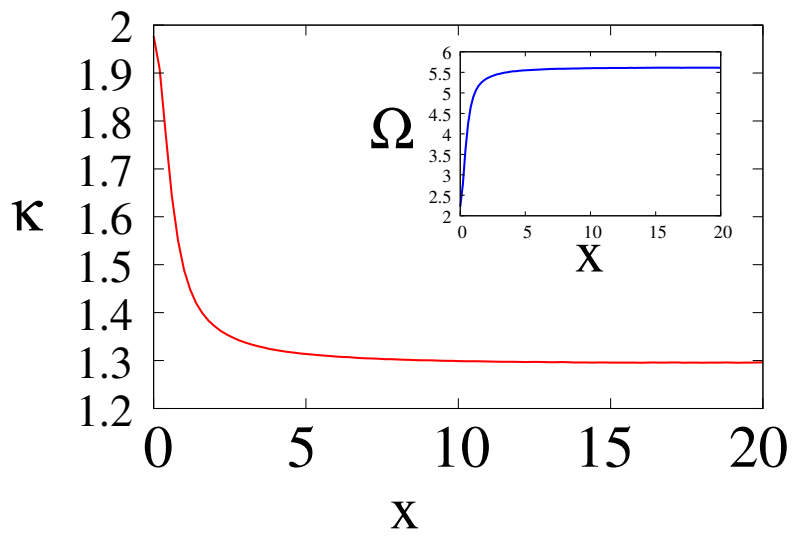

Figure 2. Evolution of $\kappa$ for the defocusing NLS equation. $(\alpha=-2, \beta=1)$. In the inset the evolution of the spectral bandwidth is shown.

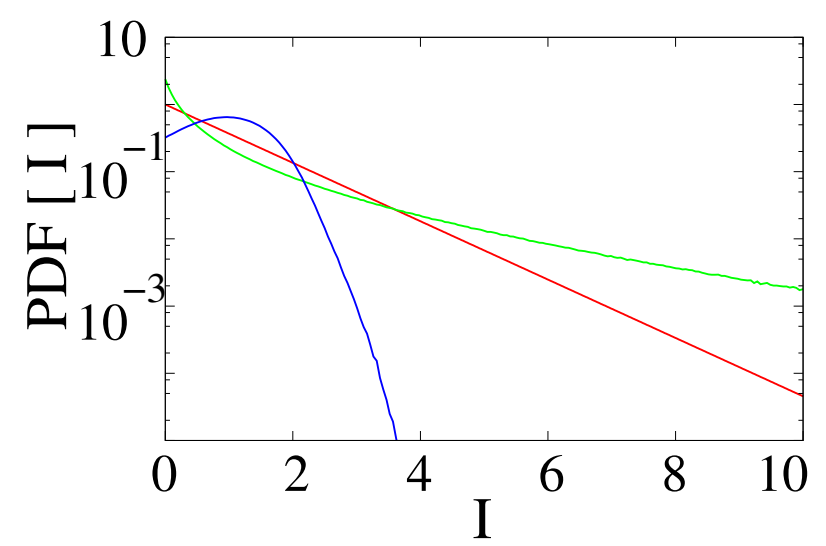

Figure 3. PDF of the normalized intensity $I=|A(t, x)|^{2} / N$ for both focusing (green line) and defocusing (blue line) NLS equation calculated for $x>20$. The exponential distribution is also shown as a red line. 


\section{Two-dimensional propagation}

We now consider the NLS in two horizontal dimensions written as an evolution equation in time:

$$
i \frac{\partial A}{\partial t}=\left(\beta \frac{\partial^{2} A}{\partial x^{2}}+\gamma \frac{\partial^{2} A}{\partial y^{2}}\right)+\alpha|A|^{2} A
$$

where

with $\gamma= \pm 1$. In the water wave context, equation (11) with $\alpha=\beta=1$ and $\gamma=-1$ arises in the deep water regime and it describes the evolution of the complex wave envelope in weakly nonlinear and narrow band (both in the direction of propagation and in the its transverse direction) approximations. The second derivative in the $y$ direction plays the role of diffraction and the equation is known as the Hyperbolic NLS. On the other hand, equation (11) with the choice of $\gamma=\beta=-1$ and $\alpha=1$, also known as the defocusing Gross-Pitaevskii equation (GPE), describes for instance the dynamics of a two-dimensional Bose-Einstein condensate. We now assume that the system in homogeneous in the domain $L_{x} \times L_{y}$ and we follow the same procedure as in the one dimensional case. Keeping in mind that now the $\kappa$ evolves in time, the same reasoning as before can be applied to get (we now assume homogeneity of the wave field):

$$
\begin{aligned}
& \kappa(t)=\kappa\left(t_{0}\right)+\frac{2}{\alpha\langle N\rangle} \times \\
& \left.\left\{\beta\left[K_{x}(t)^{2}-K_{x}\left(t_{0}\right)^{2}\right]+\gamma\left[K_{y}(t)^{2}-K_{y}\left(t_{0}\right)^{2}\right)\right]\right\},
\end{aligned}
$$

$$
1 \text { and }
$$

$$
K_{x}(t)=\sqrt{\frac{\sum_{k, l}\left\langle\left(\frac{2 \pi}{L_{x}} k\right)^{2}\left|A_{k, l}(t)\right|^{2}\right\rangle}{\sum_{k, l}\left\langle\left|A_{k, l}(t)\right|^{2}\right\rangle}},
$$

and $K_{y}(t)$ is defined in a similar fashion with the only difference that in the brackets $L_{x}$ is replaced by $L_{y}$ and $k$ with $l$.

As in the one dimensional case, we show some instructive numerical simulations of equation (11). For all cases considered, the initial condition is characterized by the following Fourier spectrum:

$$
A_{k, l}(t=0)=\sqrt{a_{0} e^{-\left(\frac{2 \pi}{L}\right)^{2} \frac{k^{2}+l^{2}}{\sigma}}} e^{i \phi_{k, l}}
$$

with $a_{0}=7.8 \times 10^{-5}, \sigma=\sqrt{10}, L=L_{x}=L_{y}=512$ and phases are taken as randomly distributed. Numerical simulations are performed with a resolution of $1024 \times 1024$ with $\Delta x=\Delta y=0.5$. To improve the statistical convergences, 10 different simulations are performed for each case with different initial random phases.

We start by considering the GPE: generally, given an initial condition localized in Fourier space, the tendency is to observe a broadening of the spectrum, thus, due to the fact that $\beta=\gamma=-1$, according to equation (12) we expect to observe a decrease of $\kappa$. Indeed, in Figure 4, we show a density plot of the two dimensional Fourier spectrum at $t=0,10,500,1000$. It is interesting to observe that the spectrum broadens isotropically and a condensate at the mode $(k, l)=(0,0)$ forms at large times (red spot in the Figure 4), see [19,20] for details. The initial value of $\kappa$, shown in Figure 5, decreases from the value of 2: starting from a spectrum characterized by random phases, extreme amplitudes are statistically not expected in the defocusing GPE. The situation is different for the hyperbolic NLS where the equation is focusing in $x$ and defocusing in the $y$ direction. Because of the opposite signs in the linear terms, we expect an initial non-isotropic evolution. Indeed, as shown in Figure 6, the spectrum evolves more rapidly in the $k_{x}$ direction, probably due to some fast evolution related to an instability of the modulational instability type, see [21]. This results in a fast increase of $\kappa$, see Figure 7 up to $t=5$. After this initial transient, the spectrum grows also in the transverse direction and the value of $\kappa$ reduces accordingly, reaching a Gaussian value. A snapshot of the intensity of the wave field taken at the time when $\kappa$ has a maximum is reported in Fig. 8: clearly, the field is characterized by the presence of a number of rogue waves embedded in an incoherent wave field.

Before concluding, we find opportune to make a comment on the evolution of the spectral bandwidth. So far, we have discussed an analytical result which provides an interesting perspective on the generation of heavy tails. However, equations (9) and (12) are not closed: an evolution equation for the spectrum is still required. The standard approach consists in considering the weakly nonlinear limit and derive the wave kinetic equation (see [22]) from the (non-integrable) NLS type of equation using the wave turbulence theory. In such an equation the linear energy is a 


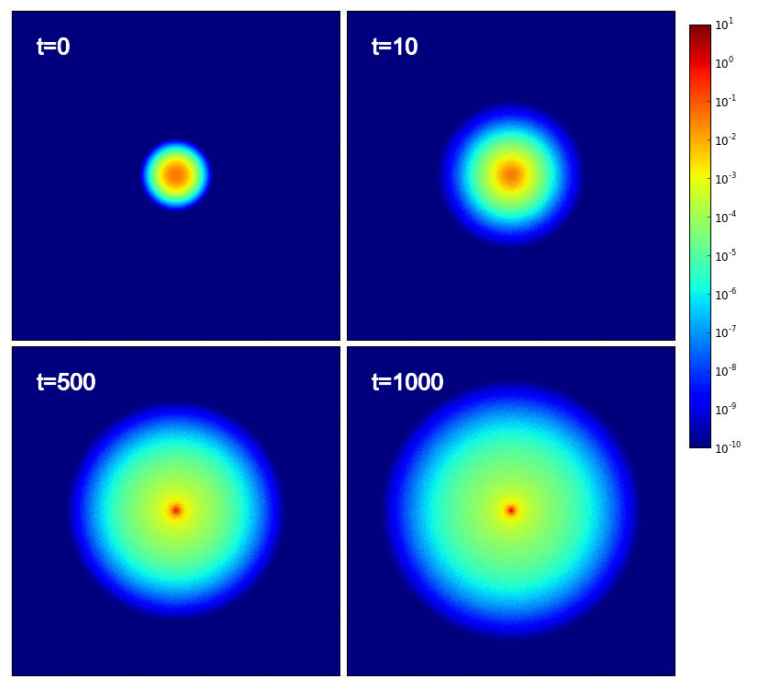

Figure 4. Evolution of the spectrum for the GPE at different times. The initial conditions are provided in equation (14)

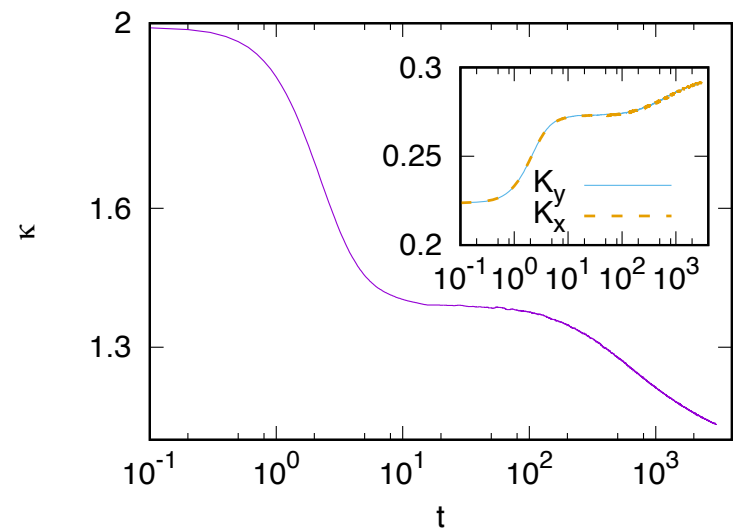

Figure 5. $\kappa$ as a function of time for numerical simulations of the GPE. The initial conditions are provided in equation (14). In the inset the evolution of the spectral bandwidth is shown. 


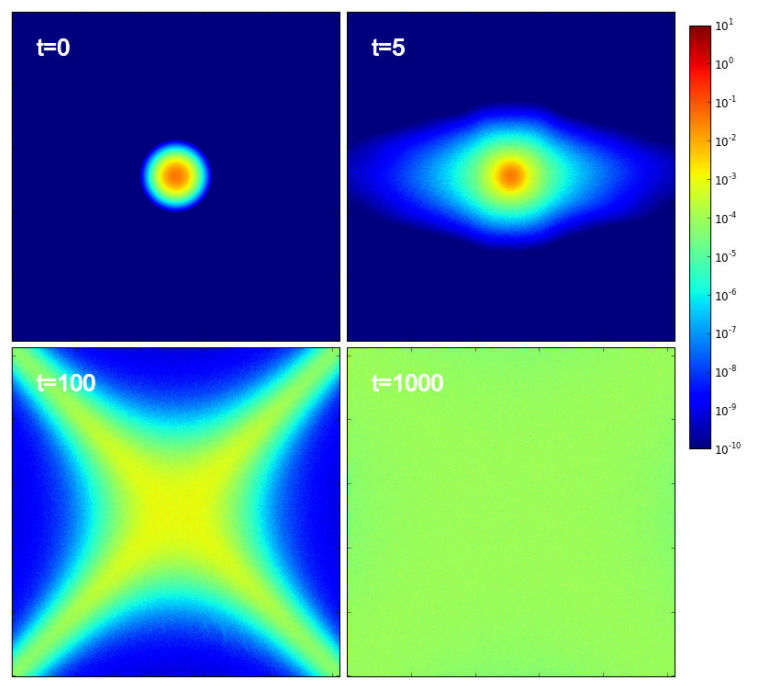

Figure 6. Evolution of the spectrum for the hyperbolic NLS equation at different times. The initial conditions are provided in equation (14)

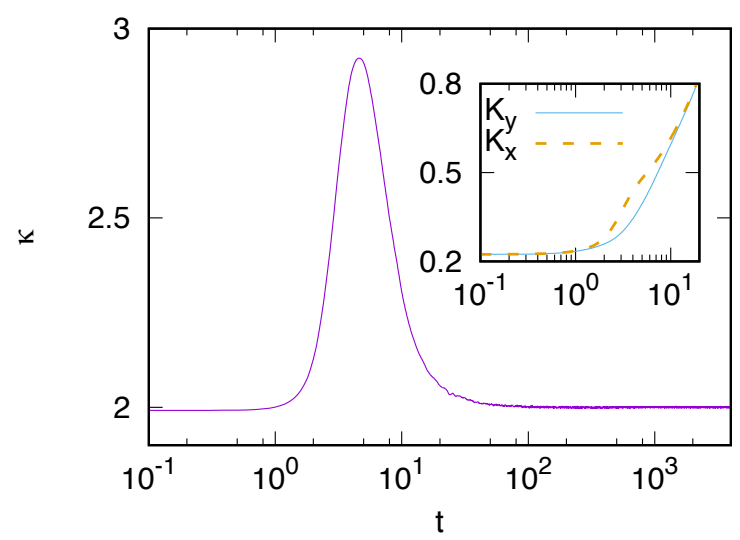

Figure 7. $\kappa(t)$ as a function of time for numerical simulations of the hyperbolic NLS. The initial conditions are provided in equation (14). In the inset, the evolution of the spectral bandwidth is shown.

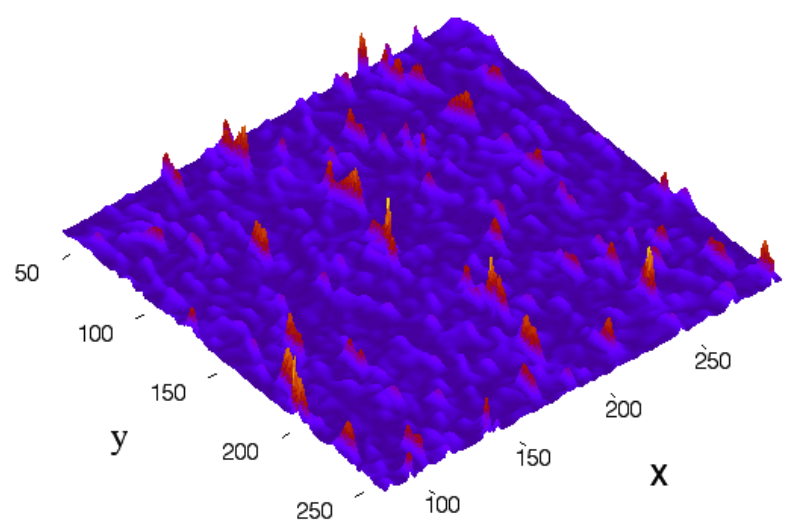

Figure 8. $|A|^{2}$ as a function of $x$ and $y$ at the time of the maximum of $\kappa$, see Fig. 7. 
constant of motion; this is a consequence of the fact that the transfer of energy and number of particle is ruled by exact resonance interactions. Therefore, even though the spectrum may evolve, the spectral bandwidth (related to the quadratic contribution to the Hamiltonian density) remains constant. Thus, if one is interested in studying changes in statistical properties of the wave field, the need of considering non-resonant interactions in the kinetic equation is essential, see $[15,23,24,25,26]$ for details on the subject.

\section{Conclusions}

In conclusion, here we have presented an identity for a class of equations characterized by the NLS nonlinearity that relates the variation of the fourth-order moment of the probability density function of the wave envelope with the variation of the spectral bandwidth. This result sheds some light on the statistical origin of rogue waves in systems described by such type of equations. It should be noted that our approach is rather general as it can be applied whenever a conserved quantity of a partial differential equation contains a moment of the distribution. For example, in the Korteweg-de Vries (KdV) equation, the Hamiltonian is directly connected to the third-order moment; therefore, given the evolution of the spectrum, a direct information on the asymmetry of the PDF for the wave displacement is available.

Acknowledgments M.O. was supported MIUR Grant PRIN 2012BFNWZ2. Dr. B. Giulinico is acknowledged for discussions. P.S. and S.R. were partially supported by the Labex CEMPI (ANR-11-LABX-0007-01) and by the French National Research Agency (ANR-12-BS04-0011 OPTIROC). The two-dimensional simulations were carried out on the High Performance Computing Cluster supported by the Research and Specialist Computing Support service at the University of East Anglia.

\section{References}

[1] S. I. Resnick, Heavy-tail phenomena: probabilistic and statistical modeling, Springer Science \& Business Media, 2007.

[2] J. Beirlant, Y. Goegebeur, J. Segers, J. Teugels, Statistics of extremes: theory and applications, John Wiley \& Sons, 2006.

[3] B. D. Malamud, Tails of natural hazards, Physics World 17 (8) (2004) 31-35.

[4] C. Kharif, E. Pelinovsky, A. Slunyaev, Rogue waves in the ocean, Springer Verlag, 2009.

[5] M. Onorato, A. R. Osborne, M. Serio, L. Cavaleri, C. Brandini, C. T. Stansberg, Observation of strongly non-gaussian statistics for random sea surface gravity waves in wave flume experiments, Phys. Rev. E 70, 067302.

[6] A.-L. Barabasi, The origin of bursts and heavy tails in human dynamics, Nature 435 (7039) (2005) $207-211$

[7] D. Solli, C. Ropers, P. Koonath, B. Jalali, Optical rogue waves, Nature 450 (7172) (2007) 1054-1057.

[8] P. Walczak, S. Randoux, P. Suret, Optical rogue waves in integrable turbulence, Physical Review Letters 114 (14) (2015) 143903.

[9] S. Toenger, T. Godin, C. Billet, F. Dias, M. Erkintalo, G. Genty, J. M. Dudley, Emergent rogue wave structures and statistics in spontaneous modulation instability, Scientific reports 5.

[10] D. Pierangeli, F. Di Mei, C. Conti, A. Agranat, E. DelRe, Spatial rogue waves in photorefractive ferroelectrics, Physical Review Letters 115 (9) (2015) 093901.

[11] S. O. Rice, Mathematical analysis of random noise, Bell Sys. Tech. J. 24 (1) (1945) 46-156.

[12] M. S. Longuet-Higgins, On the statistical distribution of the heights of sea waves, J. Marin. Res. 11 (1952) $1245-1266$.

[13] M. Onorato, S. Residori, U. Bortolozzo, A. Montina, F. Arecchi, Rogue waves and their generating mechanisms in different physical contexts, Physics Reports 528 (2) (2013) 47-89.

[14] J. M. Dudley, F. Dias, M. Erkintalo, G. Genty, Instabilities, breathers and rogue waves in optics, Nature Photonics 8 (10) (2014) $755-764$.

[15] P. A. E. M. Janssen, Nonlinear four-wave interaction and freak waves, J. Phys. Ocean. 33 (4) (2003) $863-884$.

[16] S. Randoux, P. Walczak, M. Onorato, P. Suret, Intermittency in integrable turbulence, Phys. Rev. Lett. 113 (2014) 113902. doi:10.1103/PhysRevLett.113.113902.

[17] F. Fedele, Z. Cherneva, M. Tayfun, C. Soares, Nonlinear Schr "odinger invariants and wave statistics, Physics of Fluids 22 (2010) 036601.

[18] F. Fedele, Explaining extreme waves by a theory of stochastic wave groups, Computers \& structures 85 (5) (2007) $291-303$.

[19] S. Nazarenko, M. Onorato, D. Proment, Bose-einstein condensation and berezinskii-kosterlitz-thouless transition in the two-dimensional nonlinear schrödinger model, Physical Review A 90 (1) (2014) 013624.

[20] C. Connaughton, C. Josserand, A. Picozzi, Y. Pomeau, S. Rica, Condensation of classical nonlinear waves, Physical review letters 95 (26) (2005) 263901

[21] I. E. Alber, The effects of randomness on the stability of two dimensional surface wave trains, Proc. Roy. Soc. London A636 (1978) 525-546.

[22] S. Nazarenko, Wave turbulence, Vol. 825, Springer, 2011.

[23] P. Janssen, On some consequences of the canonical transformation in the hamiltonian theory of water waves, J. Fluid Mech. 637 (1) (2009) $1-44$.

[24] S. Y. Annenkov, V. I. Shrira, Role of non-resonant interactions in the evolution of nonlinear random water wave fields, Journal of Fluid Mechanics 561 (2006) 181-207. 
[25] S. Annenkov, V. Shrira, Modelling the impact of squall on wind waves with the generalized kinetic equation, J. Phys. Ocean. 45 (3) (2015) 807-812.

[26] P. Suret, A. Picozzi, S. Randoux, Wave turbulence in integrable systems: nonlinear propagation of incoherent optical waves in single-mode fibers, Optics express 19 (18) (2011) 17852-17863. 